\title{
Influência do peso adicional no alcance manual de lactentes a termo e pré-termo: análise de variáveis categóricas do movimento ${ }^{1}$
}

\author{
Mariana Vieira da Fonseca ${ }^{a}$ (D), Ana Letícia de Souza Oliveira ${ }^{a}$, Rodrigo Luiz Carregaro ${ }^{b}$, \\ Eloisa Tudella ${ }^{c}$, Aline Martins de Toledo ${ }^{b}$ \\ ${ }^{a}$ Universidade de Brasília - UnB, Brasília, DF, Brasil.
}

'bepartamento de Fisioterapia, Universidade de Brasília - UnB, Brasília, DF, Brasil.

'Departamento de Fisioterapia, Universidade Federal de São Carlos - UFSCar, São Carlos, SP, Brasil.

\begin{abstract}
Resumo: Objetivo: Investigar o efeito do peso adicional nos ajustes proximais e distais do alcance em lactentes a termo (AT) e pré-termo tardios (PTT). Método: Foram avaliados 10 lactentes AT e 9 PTT dos 5 aos 7 meses de idade. As variáveis analisadas foram: a) ajustes proximais: unimanuais ou bimanuais; b) ajustes distais: abertura das mãos (aberta, fechada ou semiaberta) e orientação das mãos (horizontalizada, verticalizada ou oblíqua). Os procedimentos analisados foram: P1 (linha de base) e P2 (acréscimo de 20\% da massa do membro superior de peso adicional). Para verificar a associação entre os procedimentos e as variáveis dependentes utilizou-se o Teste Qui-quadrado $(p<0,05)$. Resultados: $O$ peso adicional proporcionou a diminuição de alcances bimanuais aos 5 e 7 meses no grupo PTT, o aumento da frequência de mão verticalizada e a diminuição da frequência de mão aberta no início do movimento, aos 6 meses. No grupo AT, o peso implicou no aumento da mão verticalizada no início e no fim do movimento, aos 5 meses, e ao final do movimento, aos 7 meses, e diminuiu a frequência de mão aberta no início e final do alcance, aos 6 meses. Conclusão: $O$ peso adicional favoreceu um movimento mais maduro na maioria das variáveis analisadas e, de forma mais expressiva, no grupo prematuro. Assim, pode tornar-se um instrumento para o treino inicial do alcance, quando o objetivo for a estimulação de alcances unimanuais com a mão verticalizada.
\end{abstract}

Palavras-chave: Alcance Manual, Ajustes Distais, Ajustes Proximais, Peso Adicional, Prematuridade, Lactente.

\section{Influence of additional weight in the reaching behavior of full-term and preterm infants: analysis of categorical variables of movement}

\begin{abstract}
Objective: The aim of this work is to investigate the effects of additional weight in the proximal and distal adjustments of the reaching behavior of full-term and preterm infants. Method: We evaluated 10 full-term and 9 low risk preterm infants from 5 to 7 months age. The following variables were analyzed: a) proximal adjustments: unimanual or bimanual; b) distal adjustments: hand opening (open, closed, semi-open) and hand orientation (horizontal, vertical, oblique). Variables were analyzed in two procedures: P1 (baseline) and P2 (addition of 20\% of the total mass of the infant's upper limb). To verify the association between procedures and dependent variables was applied Chi-Square Test $(p<0,05)$. Results: Additional weight decreased of bimanual frequency for the preterm group at 5 and 7 months old, increased vertical hand frequency and decreased open hand frequency at 6 months. In the full-term group, additional weight increased the vertical hand frequency at the initiation and at the end of the movement at 5 months and at the end of the movement at 7 months. It also decreased open hand frequency at the initiation and at the end of the reaching at 6 months. Conclusion: Additional weight favored a more mature movement in the majority of variables analyzed, more expressively in the preterm group. Therefore, it can become an instrument of training this skill in the beginning of the reaching, when looking for verticalized hand unimanual reaching stimulation.
\end{abstract}

Keywords: Manual Reaching, Distal Adjustments, Proximal Adjustments, Additional Weight, Prematurity, Infant.

Autor para correspondência: Mariana Vieira da Fonseca, Universidade de Brasília, Campus UnB Ceilândia, Centro Metropolitano, conjunto A, lote 01, CEP 72220-900, Brasília, DF, Brasil, e-mail: marianinhavf@gmail.com

Recebido em Ago. 11, 2017, 1ª Revisão em Maio 11, 2018, Aceito em Set. 11, 2018.

Este é um artigo publicado em acesso aberto (Open Access) sob a licença Creative Commons Attribution, que permite uso, distribuição e reprodução em qualquer meio, sem restrições desde que o trabalho original seja corretamente citado. 


\section{Introdução}

Sabe-se que as vivências cotidianas relacionadas ao meio ambiente em que o lactente e a criança estão inseridos propiciam experiências de amadurecimento de habilidades motoras durante o seu desenvolvimento (CAMPOS et al., 2017). De acordo com Ulrich (2010), protocolos terapêuticos funcionalmente relevantes devem enfatizar as atividades da vida diária, proporcionando às crianças a capacidade de moverem ativamente seus membros e corpos no espaço, e responderem a perturbaçóes realistas do ambiente, demonstrando, assim, a capacidade de se adaptarem de forma adequada. Nesse contexto, a investigação do efeito do peso adicional em habilidades motoras precoces torna-se uma relevante estratégia na tentativa de analisar as adaptaçôes dos sistemas neuromotores infantis frente a influências ambientais.

$\mathrm{O}$ uso do peso adicional tem apresentado resultados interessantes em diversas habilidades motoras precoces, como o aumento no sucesso de elevar um painel durante o movimento de chutes após a remoção imediata do peso em lactentes a termo e com Síndrome de Down (SANTOS et al., 2014); o aumento da frequência de chutes (CHEN et al., 2002); a diminuiçáo dos picos de aceleração e desaceleraçáo do braço, e o aumento da velocidade média no alcance manual em lactentes pré-termo de baixo risco (TOLEDO et al., 2012), além de proporcionar uma melhor sincronia entre os membros superiores em alcances bimanuais (ROCHA et al., 2009). Esses resultados são atribuídos principalmente ao aumento da propriocepção, da ativação neural, das unidades motoras e do recrutamento de fibras musculares, causados pelo peso adicional (CHEN et al., 2002; VAAL et al., 2002), além de favorecer a seleçâo de um padrão adaptativo mais eficiente e estável (OUT et al., 1997). Tais aspectos podem, desta forma, beneficiar o desenvolvimento de habilidades precoces de lactentes com alteraçóes neurosensoriomotoras.

$\mathrm{O}$ alcance manual, especificamente, desempenha um papel essencial no desenvolvimento geral da criança. A conquista desse marco permite uma interação dos lactentes com o ambiente de forma mais independente, o que expande suas oportunidades de coletar informações sobre objetos e aprender novos comportamentos para agir sobre estes (CORBETTA; THELEN; JOHNSON, 2000; LOBO; GALLOWAY, 2012, 2013). No entanto, limitaçóes biológicas como alteraçôes do tônus muscular, dificuldades de acoplamento da percepção-ação, dificuldades de aprendizado - podem impedir a capacidade dos lactentes de interagir com o mundo e se desenvolver a partir disso, o que tem sido observado em crianças nascidas prematuramente. Os lactentes prematuros apresentam um desempenho inferior no alcance, demonstrando um movimento com menor velocidade média e final, maior índice de ajuste, mais unidades de movimento (TOLEDO; TUDELLA, 2008), mão mais horizontalizada (DIBIASI; EINSPIELER, 2004) e maior frequência de preensão sem sucesso (TOLEDO; TUDELLA, 2008). Tais aspectos provavelmente estão relacionados a déficits de informações sensoriais e na coordenação motora e visomotora (TOLEDO; TUDELLA, 2008; GROOT, 1993; TOLEDO; SOARES; TUDELLA, 2011; HEATHCOCK; LOBO; GALLOWAY, 2008). Além disso, Fallang et al. (2005) mostraram que a ausência de alcance com sucesso aos 4 meses de idade corrigida e a baixa qualidade cinemática dois meses após o início do alcance em crianças nascidas pré-termo estão relacionadas ao desenvolvimento de uma discreta disfunção neurológica (disfunção na regulação do tônus muscular, problemas de coordenação, problemas de habilidade manipulativa, entre outros) na idade escolar. Esses achados sugerem que a presença de atrasos e distúrbios do alcance precocemente pode predizer futuros distúrbios do desenvolvimento e destacam a importância essencial de promover intervençóes motoras focadas no alcance de lactentes prematuros.

Frente a esse cenário e considerando os efeitos do peso adicional já encontrados nas variáveis cinemáticas tridimensionais do alcance, citados previamente, destaca-se a necessidade de verificar como o peso adicional influencia as demais características do movimento de alcance, a exemplo dos seus ajustes proximais (alcance uni ou bimanual) e distais (orientação e classificação da mão). $\mathrm{O}$ estudo e a análise de como o peso adicional modifica os ajustes proximais e distais de lactentes poderão contribuir para a prática clínica de fisioterapeutas e terapeutas ocupacionais, que poderão usar esse recurso terapêutico em suas intervençôes com lactentes prematuros. Além disso, a análise dessas variáveis pode ser direta e facilmente observada, não havendo a necessidade de instrumentação de alto custo para tal finalidade.

Desse modo, o objetivo do presente estudo foi investigar o efeito do peso adicional nos ajustes proximais e distais do alcance em lactentes a termo e pré-termo de 5 a 7 meses de idade.

\section{Método}

Trata-se de um estudo longitudinal com lactentes nascidos a termo e pré-termo avaliados mensalmente dos 5 aos 7 meses de vida. 
Foram selecionados por elegibilidade 147 lactentes (89 a termo e 58 pré-termo). Destes, houve uma perda amostral de 118 lactentes. Dentre os motivos para a não participação, destacam-se: $o$ não aceite dos responsáveis em participar do estudo, a ausência de contato telefônico ou o fato de os lactentes serem de outra localidade. Todos foram selecionados a partir da consulta à lista da maternidade do hospital local e recrutados por meio de contato telefônico.

Dessa forma, participaram do presente estudo 19 lactentes. Com base na idade gestacional, os lactentes foram divididos em dois grupos: A) Lactentes pré-termo tardios (PTT, $\mathrm{n}=9$ ), com idade gestacional de 34 a 36 semanas e 6 dias; B) A termo (AT, $\mathrm{n}=10$ ), com idade gestacional superior a 38 semanas. Todos os lactentes apresentaram peso ao nascer superior a 2.500 gramas.

Não foram considerados elegíveis para o estudo aqueles lactentes considerados de alto risco para lesão neurológica, tais como, 1) anoxiados; 3) más formaçôes congênitas; 4) síndromes; 5) alterações sensoriais; 6) dificuldades cardiorrespiratórias; 7) comprometimento ortopédico e 8) Apgar abaixo de 8 e 10, no primeiro e quinto minutos, respectivamente. Além disso, foram excluídos da amostra os lactentes que não participaram de pelo menos uma das avaliações realizadas.

O estudo foi desenvolvido de acordo com os preceitos éticos, tendo sido aprovado pelo Comitê de Ética institucional (parecer n. ${ }^{\circ}$ 041/2006). Os pais ou responsáveis autorizaram a participação de seus(as) filhos(as) pela assinatura do Termo de Consentimento Livre e Esclarecido (TCLE).

As avaliações foram realizadas no Laboratório de Pesquisa e Análise do Movimento (LAPAM) do Núcleo de Estudos em Neuropediatria e Motricidade (NENEM) do Departamento de Fisioterapia da Universidade Federal de São Carlos.

Os lactentes foram avaliados mensalmente do $5 .^{\circ}$ ao $7 .^{\circ}$ mês de vida. Os lactentes do grupo PTT tiveram suas idades corrigidas.

Os lactentes foram posicionados em uma cadeira infantil a $50^{\circ}$ (TOLEDO; TUDELLA, 2008), promovendo estabilidade da cabeça, tronco e quadril. A estimulação do alcance foi realizada por meio de um objeto atrativo, não sonoro, de borracha, maleável, não familiar ao lactente, com aproximadamente $05 \mathrm{~cm}$ de diâmetro e $10 \mathrm{~cm}$ de comprimento. Os lactentes foram avaliados em estado de alerta ativo ou inativo (PRECHTL; BEINTEMA, 1964), pois são os estados comportamentais em que o desempenho motor das crianças pode ser melhor avaliado, sem interferências de choro, sono ou inquietações.
As condiçóes experimentais do estudo foram:

a) Procedimento 1 (P1): no qual o objeto foi exibido pelo examinador, que estava posicionado à frente do lactente, por 2 minutos na linha média, na altura do ombro, na distância do comprimento dos braços do lactente (TOLEDO; SOARES; TUDELLA, 2011; HEATHCOCK; LOBO; GALLOWAY, 2008; DIBIASI; EINSPIELER, 2004). Após o alcance, o objeto foi cuidadosamente retirado e reapresentado a fim de eliciar um novo alcance. $\mathrm{O}$ número total de alcances dependeu de cada lactente (DIBIASI; EINSPIELER, 2004);

b) Procedimento 2 (P2) - Peso Adicional: as condiçóes experimentais deste procedimento foram similares ao Procedimento 1; no entanto, foi adicionado, em ambos os punhos, um bracelete com peso correspondente a $20 \%$ da massa total do membro do lactente, obtida por uma equação de regressão (ZERNICKE; SCHNEIDER, 1992).

A escolha do acréscimo de $20 \%$ da massa do membro superior ocorreu devido aos benefícios já encontrados na literatura com essa porcentagem de peso no alcance de lactentes típicos (OUT et al., 1997). Além disso, o uso de menores porcentagens não apresentou resultados significantes na cinemática de lactentes típicos (ROCHA et al., 2009; VAN DER FITS; HADDERS-ALGRA, 1998).

Todos os procedimentos foram filmados utilizando-se uma câmera digital, de $60 \mathrm{~Hz}$, acoplada a um tripé, posicionada póstero-superiormente à cadeira, de modo que os membros superiores fossem visíveis ao longo de toda a trajetória do alcance. As imagens foram analisadas por meio do Sistema Dvideow, o qual permite verificar o movimento quadro a quadro.

O alcance foi excluído quando o lactente apresentou choro ou irritação durante o movimento e quando o lactente iniciou o movimento do braço com a máo próxima ao objeto (KONCZAK; DICHGANS, 1997).

Foram considerados dois momentos para análise do posicionamento da máo do lactente: a) Momento 1: início do alcance, considerado como o quadro que mostrou o primeiro movimento de um ou ambos os membros superiores em direção ao objeto; b) Momento 2: no toque do objeto, considerado como o quadro no qual qualquer parte da mão do lactente tocou o objeto.

A partir da imagem registrada em ambos os momentos, foi possível avaliar as variáveis categóricas: 
alcance uni ou bimanual (ajustes proximais), classificação e orientação das mãos (ajustes distais).

Nos ajustes proximais, foi considerada a iniciativa de direcionar um ou ambos os membros superiores ao alvo apresentado, sendo classificados em:

a) Ajuste Unimanual: quando o lactente deslocou somente um dos membros superiores em direção ao alvo, até tocá-lo (CORBETTA; THELEN; JOHNSON, 2000; CORBETTA; THELEN, 1996);

b) Ajuste Bimanual: quando o lactente estendeu simultaneamente os membros superiores em direção ao alvo (CORBETTA; THELEN; JOHNSON, 2000) ou quando os membros superiores saíram da posição inicial com atraso igual ou inferior a 20 quadros de uma mão em relação à outra (TOLEDO; SOARES; TUDELLA, 2011).

Consideraram-se, na análise dos ajustes distais, os diferentes posicionamentos de mãos e dedos, analisados em dois momentos: no início do movimento e no toque do objeto. Foram avaliados os seguintes ajustes distais:

a) Orientações da Palma da Mão: foram classificadas em: horizontal - quando o antebraço estivesse em pronação, com a palma da mão voltada para baixo; vertical - quando o antebraço estivesse em posição neutra e a palma da mão orientada para a linha média do corpo do lactente, e oblíqua - quando a máo estivesse em posiçấo intermediária em relação às outras duas supracitadas (TOLEDO; SOARES; TUDELLA, 2011; FAGARD, 2000);

b) Abertura da mão: Foi considerada mão aberta, quando as articulaçóes metacarpofalangeanas e interfalangeanas estivessem estendidas; mãos fechadas, quando as articulações metacarpofalangeanas e interfalangeanas estivessem fletidas, e mão semiaberta, quando as articulaçóes metacarpofalangeanas estivessem fletidas, enquanto as articulaçôes interfalangeanas estivessem estendidas, ou vice-versa (TOLEDO; SOARES; TUDELLA, 2011).

Para a análise dos dados, utilizou-se o Programa SPSS versão 17.0. Utilizou-se o Teste Qui-quadrado para verificar a associação dos ajustes proximais e distais com o procedimento (linha de base e peso adicional) em cada grupo, separadamente. A significância adotada foi de 5\% $(p<0,05)$. Seguindo a análise previamente mencionada, realizamos o cálculo do poder do teste na variável primária (ajuste proximal) dos lactentes prematuros. Verificamos um poder de $85 \%$, quando comparamos a linha de base e o peso adicional na idade de 5 meses (com tamanho do efeito $\mathrm{w}=0,22$ ), e um poder de $99 \%$ aos 7 meses (com tamanho do efeito $\mathrm{w}=0,62$ ).

\section{Resultados}

Foram coletados 1.071 alcances, sendo destes 931 analisados e 140 excluídos. A frequência de alcances analisados em cada grupo, assim como a dos excluídos das análises, estáo demonstradas na Tabela 1. A descrição da média de peso acrescida nos braceletes em cada grupo e nas diferentes idades avaliadas está indicada na Tabela 2.

\subsection{Ajustes proximais}

Verificou-se que o peso diminuiu a frequência de alcances bimanuais e aumentou a frequência de unimanuais aos 5 e 7 meses $\left(x^{2}=9,8210, p=0,0179\right.$ $\mathrm{e}^{\chi^{2}}=8,1861, p=0,0167$, respectivamente), no grupo PTT (Tabela 3). Para o grupo AT, o peso não gerou influências significantes na frequência de alcances $(p<0,05)$.

\subsection{Orientação da mão}

Verificou-se que, no grupo AT, no início do movimento, o peso aumentou a frequência de mão verticalizada e diminuiu a mão horizontalizada aos

Tabela 1. Número de alcances analisados e excluídos em cada grupo (PTT - prematuro e AT - a termo) e em cada procedimento (P1 - linha de base e $\mathrm{P} 2$ - peso adicional).

\begin{tabular}{ccccc}
\hline \multirow{2}{*}{ Grupo } & \multicolumn{2}{c}{$\begin{array}{c}\text { Alcances } \\
\text { analisados }\end{array}$} & \multicolumn{2}{c}{$\begin{array}{c}\text { Alcances } \\
\text { excluídos* }\end{array}$} \\
\cline { 2 - 5 } & P1 & P2 & P1 & P2 \\
\hline PTT & 231 & 224 & 42 & 32 \\
AT & 229 & 247 & 36 & 30 \\
Total & 460 & 471 & 78 & 62 \\
\hline
\end{tabular}

*As exclusões ocorreram devido a choro, irritação e início do alcance com a mão próxima do objeto.

Tabela 2. Média e desvio padrão $( \pm)$ do peso acrescido em gramas nos braceletes, em cada grupo e em cada idade analisada.

\begin{tabular}{crcc}
\hline Grupo & \multicolumn{1}{c}{ 5 meses } & 6 meses & $\mathbf{7}$ meses \\
\hline PTT & $73,90 \pm 9,3$ & $78,9 \pm 8,1$ & $82,9 \pm 8,0$ \\
AT & $74,7 \pm 9,1$ & $81,3 \pm 7,7$ & $85,3 \pm 7,8$ \\
\hline
\end{tabular}


5 meses $\left[{ }_{\mathrm{X}}{ }^{2}(2)=9,8210, p=0,0436\right]$. Aos 6 meses, observou-se aumento da frequência da mão verticalizada $\left[_{\mathrm{X}}{ }^{2}(2)=12,0285, p=0,0171\right]$; e, aos 7 meses, houve aumento da máo verticalizada e diminuiçáo da mão horizontalizada $\left[{ }_{\mathrm{X}}{ }^{2}(2)=18,9645, p=0,0008\right]$ (Tabela 3 ). Ao final do movimento, o peso aumentou a frequência de mão verticalizada aos 5 meses $\left[_{\mathrm{x}}^{2}(2)=12,9732\right.$, $p=0,0114]$. Aos 7 meses, houve aumento da mão verticalizada e diminuição da mão horizontalizada com o uso do peso $\left[_{X}^{2}(2)=28,2144, p<0,0001\right]$ (Tabela 4).

No grupo PTT, observou-se que o peso aumentou a frequência de mão verticalizada e diminuição da mão horizontalizada no início do movimento aos 6 meses $\left[_{\mathrm{X}}{ }^{2}(2)=16,3408, p=0,0026\right]$ (Tabela 4).

\subsection{Classificação da mão}

Verificou-se que, no AT, o peso diminuiu a frequência de mão aberta no início do movimento de alcance aos 6 meses $\left[{ }_{\mathrm{x}}{ }^{2}(2)=6,8654, p=0,0323\right]$ e 7 meses $\left[{ }_{\mathrm{x}}^{2}(2)=7,7460, p=0,0208\right]$; e, no final do movimento, também aos $6\left[_{\mathrm{x}}^{2}(2)=7,1996\right.$, $p=0,0273]$ e 7 meses $\left[{ }_{x}{ }^{2}(2)=28,2198, p<0,0001\right]$ (Tabela 5).

Tabela 3. Frequência (\%) dos alcances unimanuais e bimanuais no início e no final do movimento nas idades de 5 a 7 meses, considerando ambos os procedimentos (P1 e P2) e ambos os grupos (PT'T - prematuro e AT - a termo).

\begin{tabular}{|c|c|c|c|c|c|c|c|c|}
\hline & \multicolumn{8}{|c|}{ Grupo } \\
\hline & \multicolumn{4}{|c|}{ AT } & \multicolumn{4}{|c|}{ PTT } \\
\hline & \multicolumn{2}{|c|}{ Unimanual } & \multicolumn{2}{|c|}{ Bimanual } & \multicolumn{2}{|c|}{ Unimanual } & \multicolumn{2}{|c|}{ Bimanual } \\
\hline & P1 & P2 & P1 & P2 & P1 & P2 & P1 & P2 \\
\hline 5 meses & 46,4 & 53,6 & 54,5 & 45,5 & 35,8 & $64,2^{*}$ & 73 & $27 *$ \\
\hline 6 meses & 47,2 & 52,8 & 64 & 36 & 46,2 & 53,8 & 50 & 50 \\
\hline 7 meses & 47,3 & 52,7 & 41,6 & 58,4 & 45 & $65^{*}$ & 66,6 & $33,4^{*}$ \\
\hline
\end{tabular}

Tabela 4. Frequência (\%) da orientação da mão (horizontalizada, oblíqua e verticalizada) no início e no final do movimento nas idades de 5 a 7 meses, considerando ambos os procedimentos (P1 e P2) e ambos os grupos (PTT - prematuro e AT - a termo).

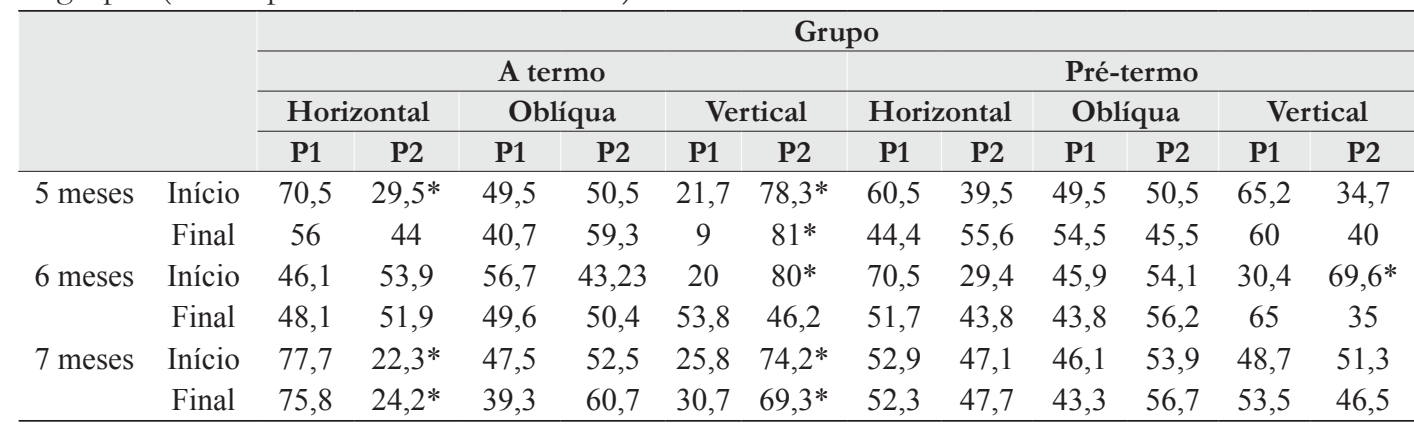

${ }^{*} p<0,05$ - Diferença significativa entre os procedimentos em cada idade e em cada grupo.

Tabela 5. Frequência (\%) da classificação da mão (aberta e semiaberta) no início e no final do movimento nas idades de 5 a 7 meses, considerando ambos os procedimentos (P1 e P2) e ambos os grupos (PTT - prematuro e AT - a termo).

\begin{tabular}{|c|c|c|c|c|c|c|c|c|c|}
\hline & & \multicolumn{8}{|c|}{ Grupo } \\
\hline & & \multicolumn{4}{|c|}{ AT } & \multicolumn{4}{|c|}{ PTT } \\
\hline & & \multicolumn{2}{|c|}{ Aberta } & \multicolumn{2}{|c|}{ Semiaberta } & \multicolumn{2}{|c|}{ Aberta } & \multicolumn{2}{|c|}{ Semiaberta } \\
\hline & & P1 & P2 & P1 & P2 & P1 & P2 & P1 & P2 \\
\hline \multirow{2}{*}{5 meses } & Início & 38 & 62 & 48,2 & 51,8 & 0 & 0 & 54,3 & 45,7 \\
\hline & Final & 30,7 & 69,3 & 49,2 & 50,8 & 60 & 40 & 53,7 & 46,3 \\
\hline \multirow[t]{2}{*}{6 meses } & Início & 83,4 & $16,6^{*}$ & 50,9 & 49,1 & 100 & $0^{*}$ & 44,3 & 55,7 \\
\hline & Final & $71,5^{*}$ & $28,5^{*}$ & 52,7 & 47,3 & 70,4 & $29,6^{*}$ & 37 & 63 \\
\hline \multirow[t]{2}{*}{7 meses } & Início & $75^{*}$ & $25^{*}$ & 45,4 & 54,6 & 60 & 40 & 46,4 & 63,6 \\
\hline & Final & $72,7^{*}$ & $27,3^{*}$ & 40,5 & 59,5 & 73,4 & $26,6^{*}$ & 34,7 & 65,3 \\
\hline
\end{tabular}

*p $<0,05$ - Comparação entre os procedimentos em cada idade e em cada grupo. 
No grupo PTT, observou-se que o peso diminuiu a frequência de mão aberta no início do movimento de alcance aos 6 meses $\left[{ }_{x}^{2}(2)=11,2910, p=0,0035\right]$ e no final do movimento aos $6\left[_{\mathrm{x}}{ }^{2}(2)=45,5740\right.$, $p<0,0001]$ e 7 meses $\left[{ }_{X}{ }^{2}(2)=38,6596, p<0,0001\right]$ (Tabela 5).

Em ambos os grupos, não foram observados alcances com a mão fechada, não sendo apresentada essa categoria na Tabela 5.

\section{Discussão}

O presente estudo investigou o efeito do peso adicional nas variáveis categóricas do alcance em lactentes a termo e pré-termo dos 5 aos 7 meses de idade. De uma forma geral, pode-se observar que o peso adicional favoreceu os ajustes proximais, diminuindo os alcances bimanuais e aumentando os alcances unimanuais aos 5 e 7 meses, além de ter aumentado a frequência de máo verticalizada aos 6 meses, no grupo PTT. Observou-se ainda, no PTT, uma diminuição da frequência de mão aberta aos 6 e 7 meses. No grupo AT, o peso causou um aumento da frequência de máo verticalizada e diminuição da mão horizontalizada na faixa etária de 5 a 7 meses, além de ter diminuído a frequência de mão aberta aos 6 meses.

Especificamente com relação aos ajustes proximais, pode-se observar que o peso adicional influenciou tais ajustes nos lactentes prematuros, favorecendo a realização de alcances unimanuais. Está bem definido na literatura que o desenvolvimento dos ajustes proximais em lactentes típicos acontece inicialmente com predomínio de movimentos bimanuais, progredindo em seu amadurecimento para alcances unimanuais, por volta dos 6 meses (FAGARD; LOCKMAN, 2005; VAN HOF et al., 2005). Dessa forma, o uso do peso nestes lactentes contribuiu para a realização de um movimento cinesiológico mais maduro e próximo das características de um alcance típico na idade de 6 meses.

A literatura associa a utilização do peso adicional à estimulação de receptores de monitorização do esforço muscular, como o órgáo tendinoso de Golgi (MASSION, 1998), além de associá-lo a um aumento da propriocepção na articulação (CHEN et al., 2002). Além disso, de acordo com Silva, Rocha e Tudella (2011), os alcances unimanuais são realizados com o membro superior mais habilidoso e proporcionam um menor gasto energético. Podemos, dessa forma, afirmar que o peso de $20 \%$ da massa do membro foi suficiente para estimular os receptores de esforço muscular e propriocepçáo, e, assim, os lactentes prematuros possivelmente usaram o alcance unimanual como uma estratégia para diminuir o gasto energético provocado pela sobrecarga imposta.

$\mathrm{O}$ fato de ter ocorrido a influência do peso adicional somente nos lactentes prematuros pode estar relacionado às características intrínsecas nesta população, principalmente aquelas relacionadas ao controle postural. Sabe-se que lactentes prematuros de baixo risco apresentam uma extensão de tronco mais acentuada (GROOT et al., 1992) e um aumento da atividade muscular postural durante o movimento de alcance, refletindo uma maior necessidade de estratégias de organização da postura para a realização da tarefa (VAN DER FITS; HADDERS-ALGRA, 1999). Verifica-se também que os ajustes posturais dos lactentes prematuros são desorganizados temporalmente e apresentam déficit de modulação para condições específicas da tarefa do alcance (VAN DER FITS; HADDERS-ALGRA, 1999), além de apresentarem uma pior qualidade das atividades de membro superior quando comparados a lactentes a termo (PLANTINGA; PERDOCK; GROOT, 1997). Considerando as alterações posturais dos lactentes prematuros, além dos ajustes posturais compensatórios necessários para levar ambos os membros contra a gravidade, é possível afirmar que os lactentes prematuros adaptaram seu sistema, aumentando os alcances unimanuais como estratégia para compensar as demandas impostas pelo peso adicional.

Com relação à orientação da mão, verificamos que o peso favoreceu a verticalização da máo, principalmente nos lactentes a termo, na faixa etária estudada.

Um dos fatores que podem ter contribuído para a verticalização da mão na condição com peso é a ação do músculo bíceps braquial. De acordo com Bakker et al. (2010), o bíceps braquial é o músculo motor primário durante o alcance manual em lactentes a termo de 4 a 6 meses de idade. Desta forma, para vencer o peso adicional, conclui-se que possivelmente ocorreu um maior recrutamento de fibras musculares dessa musculatura. Entretanto, a ação do bíceps é prejudicada em situações de pronação de antebraço e, para garantir uma melhor ação do sistema em resposta à carga com vantagem biomecânica, utiliza-se a ação supinadora do bíceps (SMITH; WEISS; LEHMKUHL, 1997). Dessa forma, podemos afirmar que o peso de $20 \%$ da massa foi suficiente para que o lactente modificasse a orientaçáo da mão da posição horizontal (ou pronada) para uma posição verticalizada (com maior supinação), favorecendo o desempenho funcional do alcance. 
Observamos que o aumento da verticalização da mão ocorreu de forma mais expressiva nos lactentes a termo. Este resultado se deve, possivelmente, ao fato de os lactentes prematuros apresentarem maior frequência de mão horizontalizada nesta faixa etária que lactentes a termo, como observado no estudo de Heathcock, Lobo e Galloway (2008). Além disso, a literatura aponta que os lactentes nascidos prematuros apresentam um tônus muscular mais baixo que os lactentes nascidos a termo (MCGREW et al., 1985). Dessa forma, podemos inferir que a soma desses dois fatores (o tônus diminuído e a posição do membro superior adotada) pode ter dificultado a execução do movimento de supinação do antebraço para essa população, dificultando o aumento da ativação muscular para o posicionamento da mão verticalizada. Tal resultado sugere, portanto, que as informaçóes proprioceptivas oferecidas pelo peso adicional não foram suficientes para alterar o posicionamento do antebraço, nesses lactentes.

Com relaçáo à classificação da mâo, foi observado que o peso adicional diminuiu a frequência de mão aberta em ambos os grupos. Este resultado pode estar relacionado ao fato do peso adicional requerer diferentes tipos de contraçôes musculares durante o movimento de alcance (TOLEDO; SOARES; TUDELLA, 2011). De acordo com Smith, Weiss e Lehmkuhl (1997), para realizar o alcance com uma carga no ponto mais distal do antebraço, o lactente precisa controlar a flexão de ombro e a extensão do cotovelo, realizando uma contração excêntrica do bíceps para manter o braço estendido. Concomitantemente, uma contraçáo concêntrica dos músculos extensores de dedos é realizada para abrir a mão e, em seguida, realiza-se uma contração concêntrica dos músculos intrínsecos da mão e flexores de punho e dos dedos, para realizar a preensão (SMITH; WEISS; LEHMKUHL, 1997). A necessidade de controlar diferentes grupos musculares com uma sobrecarga do peso adicional parece ter desfavorecido a abertura dos dedos. Neste caso, a sinergia muscular pode ter sido prejudicada e os lactentes, dessa forma, não conseguiram manter a abertura da mão com o peso adicional. Vale ressaltar, no entanto, que a avaliação com o peso só ocorreu em um determinado momento, durante 2 minutos. Assim, os resultados aqui apresentados representam seu efeito de forma aguda. Não é possível concluir, portanto, se o uso prolongado do peso poderá aumentar o nível de habituação proprioceptiva e de contração muscular, favorecendo uma melhor adaptação ao peso e um aumento da frequência da mão aberta.

Os resultados do presente estudo contribuirão para a prática clínica de fisioterapeutas e terapeutas ocupacionais que atuam na reabilitação de lactentes prematuros. A utilização do peso adicional como recurso terapêutico poderá ocorrer principalmente quando o foco terapêutico for a realizaçáo de alcances unimanuais e o posicionamento vertical da mão, ao realizar o toque no objeto. Apesar de os resultados do presente estudo se referirem a uma única avaliação, é possível extrapolar a utilização desse recurso como ferramenta útil e de fácil implementação na prática clínica de terapeutas ocupacionais e fisioterapeutas. No entanto, faz-se necessária a realizaçáo de novos estudos para avaliar o uso prolongado do peso adicional em treinos funcionais, em diversas variáveis do alcance.

As limitaçóes deste estudo compreendem o número reduzido de participantes, apesar do alto poder do teste comprovado estatisticamente, fato que nos aponta a necessidade de cautela na generalização dos resultados. No entanto, os resultados podem ser considerados achados preliminares que servirão de base para a realizaçáo de novos estudos com o uso do peso adicional como treinamento funcional, o que poderia mudar consideravelmente os resultados apresentados.

\section{Conclusão}

O presente estudo demonstrou que o peso adicional alterou a dinâmica das variáveis categóricas do alcance, favorecendo um movimento mais maduro representado pelo aumento de alcance unimanual e com a mão verticalizada, em ambos os grupos; observou-se, no entanto, que tal alteração da dinâmica ocorreu de maneira mais expressiva nos lactentes prematuros. $\mathrm{O}$ peso adicional pode se tornar um instrumento para o treino inicial do alcance, quando se objetiva a exploração da habilidade com características biomecânicas mais favoráveis.

\section{Referências}

BAKKER, H. et al. Development of proximal arm muscle control during reaching in young infants: From variation to selection. Infant Behavior \& Development, Norwood, v. 33, n. 1, p. 30-38, 2010.

CAMPOS, S. D. F. et al. O brincar para o desenvolvimento do esquema corporal, orientação espacial e temporal: análise de uma intervenção. Cadernos Brasileiros de Terapia Ocupacional, São Carlos, v. 25, n. 2, p. 275-285, 2017.

CHEN, Y. P. et al. Making the mobile move: constraining task and environment. Infant Behavior \& Development, Norwood, v. 25, n. 2, p. 195-220, 2002.

CORBETTA, D.; THELEN, E. The developmental origins of bimanual coordination: a dynamic perspective. 
Journal of Experimental Psychology, Human Perception and Performance, Washington, v. 22, n. 2, p. 502-522, 1996.

CORBETTA, D.; THELEN, E.; JOHNSON, K. Motor constraints on the development of perception-action matching in infant reaching. Infant Behavior \& Development, Norwood, v. 23, n. 3/4, p. 351-374, 2000.

DIBIASI, J.; EINSPIELER, C. Load perturbation does not influence spontaneous movements in 3-month-old infants. Early Human Development, Limerick, v. 77, n. 1/2, p. 37-46, 2004.

FAGARD, J. Linked proximal and distal changes in the reaching behavior of 5-to 12 month-old human infants grasping objects of different sizes. Infant Behavior \& Development, Norwood, v. 23, n. 3/4, p. 317-329, 2000.

FAGARD, J.; LOCKMAN, J. The effect of task constraints on infants' (bi)manual strategy for grasping and exploring objects. Infant Behavior \& Development, Norwood, v. 28, n. 3, p. 305-315, 2005.

FALLANG, B. et al. Quality of reaching and postural control in young preterm infants is related to neuromotor outcome at 6 years. Pediatric Research, New York, v. 58, n. 2, p. 347-353, 2005.

GROOT, L. et al. Development of the relationship between active and passive muscle power in preterms after term age. Neuropediatrics, Stuttgart, v. 23, n. 6, p. 298-305, 1992.

GROOT, L. L'intervention precoce et la stimulation de developpement chez l'enfant premature. Une mode ou une necessité? Motricité Cerebrale, Paris, v. 14, p. 10-16, 1993.

HEATHCOCK, J. C.; LOBO, M.; GALLOWAY, C. J. Movement training advances the emergence of reaching in infants born at less than 33 weeks of gestational age: a randomized clinical trial. Physical Therapy, New York, v. 88, n. 3, p. 310-322, 2008.

KONCZAK, J.; DICHGANS, J. The development toward stereotypic arm kinematics during reaching in the first 3 years of life. Experimental Brain Research, Berlin, v. 117, n. 2, p. 346-354, 1997.

LOBO, M. A.; GALLOWAY, J. C. Enhanced handling and positioning in early infancy advances development throughout the first year. Child Development, Malden, v. 83, n. 4, p. 1290-1302, 2012.

LOBO, M. A.; GALLOWAY, J. C. The onset of reaching significantly impacts how infants explore both objects and their bodies. Infant Behavior \& Development, Norwood, v. 36, n. 1, p. 14-24, 2013.

MASSION, J. Postural control systems in developmental perspective. Neuroscience and Biobehavioral Reviews, New York, v. 22, n. 4, p. 465-472, 1998.

MCGREW, L.; CATLIN, P. A.; BRIDGFORD, J. The landau réaction in fullterm and prétérm infants at four months of age. Developmental Medicine \& Child Neurology, Oxford, v. 27, n. 2, p. 161-169, 1985.

OUT, L. et al. The effect of posture on early reaching movement. Journal of Motor Behavior, New York, v. 30, n. 3, p. 260-272, 1997.
PLANTINGA, Y.; PERDOCK, J.; GROOT, L. Hand function in low-risk preterm infants: its relation to muscle power regulation. Developmental Medicine \& Child Neurology, Oxford, v. 39, n. 1, p. 6-11, 1997.

PRECHTL, H. F. R.; BEINTEMA, D. J. The neurological examination of the full-term newborn infant. Archives of Disease in Childhood, London, v. 39, n. 207, p. 590-591, 1964.

ROCHA, N. A. C. F. et al. The effect of additional weight load on infant reaching. Infant Behavior \& Development, Norwood, v. 32, n. 2, p. 234-237, 2009.

SANTOS, G. L. et al. Influence of additional weight on the frequency of kicks in infants with Down syndrome and infants with typical development. Brazilian Journal of Physical Therapy, São Carlos, v. 18, n. 3, p. 237-246, 2014.

SILVA, F. P. S.; ROCHA, N. A. C. F.; TUDELLA, E. Can size and rigidity of objects influence infant's proximal and distal adjustments of reaching? Revista Brasileira de Fisioterapia, São Carlos, v. 15, n. 1, p. 37-44, 2011.

SMITH, L. K.; WEISS, E. L.; LEHMKUHL, L. D. Cinesiologia Clinica de Brunnstrom. São Paulo: Editora Manole, 1997.

TOlEDO, A. M.; SOARES, D. A.; TUDELlA, E. Additional weight influences the reaching behavior of low-risk preterm infants. Journal of Motor Behavior, New York, v. 44, n. 3, p. 203-212, 2012.

TOLEDO, A. M.; SOARES, D. A.; TUDELlA, E. Proximal and distal adjustments of reaching behavior in preterm infants. Journal of Motor Behavior, New York, v. 43, n. 2, p. 137-145, 2011.

TOLEDO, A. M.; TUDELLA, E. The development of reaching behavior in low-risk preterm infants. Infant Behavior \& Development, Norwood, v. 31, n. 3, p. 398-407, 2008.

ULRICH, B. D. Opportunities for early intervention based on theory basic neuroscience, and clinical science. Physical Therapy, New York, v. 90, n. 12, p. 1868-1880, 2010.

VAAL, J. et al. Spontaneous leg movements in infants with and without periventricular leukomalacia: effects of unilateral weighting. Behavioural Brain Research, Amsterdam, v. 129, n. 1/2, p. 83-92, 2002.

VAN DER FITS, I. B. M.; HADDERS-ALGRA, M. The development of postural responses patterns during reaching in healthy infants. Neuroscience and Biobehavioral, New York, v. 22, n. 4, p. 521-526, 1998.

VAN DER FITS, I. B. M.; HADDERS-ALGRA, M. Development of postural adjustments during reaching in preterm infants. Pediatric Research, New York, v. 46, n. 1 , p. $1-7,1999$.

VAN HOF, P. et al. The confluence of intrinsic and extrinsic constraints on 3- to-9-month-old infants catching behavior. Infant Behavior \& Development, Norwood, v. 28, n. 2, p. 179-193, 2005.

ZERNICKE, R. F.; SCHNEIDER, K. Biomechanics and developmental neuromotor control. Child Development, Malden, v. 64, n. 4, p. 982-1004, 1992. 


\section{Contribuição dos Autores}

Todos os autores participaram da redação do artigo, análise e discussão dos dados e aprovaram a versão final do texto.

\section{Fonte de Financiamento}

Este estudo foi apoiado pela Fundação de Apoio à Pesquisa do Estado de São Paulo (FAPESP), processo número: 06/50768-9.

\section{Notas}

${ }^{1}$ Este trabalho faz parte de uma pesquisa e todos os princípios éticos foram seguidos, sendo aprovado pelo Comitê de Ética institucional da Universidade Federal de São Carlos (parecer n. ${ }^{o}$ 041/2006). 